\author{
Military Technical College \\ Kobry El-Kobbah, \\ Cairo, Egypt
}

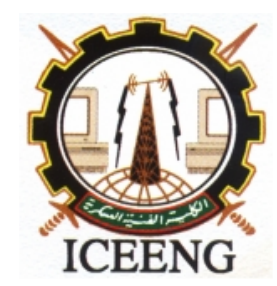

\title{
Security constrained optimal dispatch using ant colony optimization algorithm
}

\author{
By
}
A. A. Abou El-Ela*
A. M. Kinawy*
R. A. El-Sehiemy**
M. T. Mouwafi*

\section{$\underline{\text { Abstract: }}$}

This paper proposes a procedure for solving a security constrained optimal dispatch (SCOD) problem under normal and emergency conditions using ant colony optimization (ACO) algorithm. The objective function is to minimize the non-linear generation cost function by optimizing the settings the real power generation outputs under equality and inequality constraints. The proposed algorithm is applied to 5-bus system, IEEE 14-bus and IEEE 30-bus test systems. An application of the proposed algorithm to the west Delta network (WDN) as a part of the Unified Egyptian Network (UEN) considering the valve-points effects has been demonstrated. Numerical results are compared with those obtained using conventional optimization techniques as linear programming (LP), fuzzy linear programming (FLP) technique and genetic algorithm (GA). Simulation results show that the proposed ACO algorithm for the SCOD is more accurate and efficient, especially with increasing the system size.

\section{Keywords:}

Optimal dispatch, security, ant colony optimization algorithm, valve-points

* College of Engineering, Minoufiya University, Minoufiya, Egypt

** College of Engineering, Kafrelsehiekh University, Kafrelsehiekh, Egypt 


\section{Introduction:}

Security constrained optimal dispatch (SCOD) is one of the optimization problems in power systems that optimally allocate the power demand among the committed generators in economical manner while satisfying system security constraints. The problem becomes more complicated due to the non-linear nature of the objective function and constraints of real life problems. The objective function may be convex or nonconvex based on the characteristic of the supply. Different optimization methods were used for solving the economic dispatch (ED) problem. Madrigal and Quintana [1] presented an analytical solution to the classic economic dispatch problem using duality theory to derive an expression to compute both the exact primal (dispatches) and dual (marginal cost) solution to the problem without the need for numerical iterative optimization algorithms. No conflict of interest is caused if the (ED) model is used as an optimization-based electricity auction. C. Chen and N. Chen [2] solved the economic dispatch problem considering transmission capacity constraints using direct search method (DSM) to handle a number of inequality and equality constraints and units with any kind of fuel cost functions. For improving the performance of direct search procedure, a novel strategy with multi-level convergence is incorporated in the DSM to minimize the number of total iterations in the searching process.

Pathom et al. [3] presented a methodology for solving the dynamic economic dispatch (DED) problem using evolutionary programming (EP) combined with sequential quadratic programming (SQP) that consists of two parts. The first part employed the property of EP which can provide a near global search region at the beginning. When the specified termination criteria of EP are reached, the local search SQP is applied to tune the control variables to obtain the final optimal solution. Qing et al. [4] proposed a novel approach based on the analysis of the process of solution of ED problem by Lagrangian Relaxation, called dynamic queuing (DQ) algorithm.

Xihui and Quintana [5] presented an improving an interior-point based OPF by a predictor-corrector primal-dual log-barrier (PCPDLB) method as a sequence of linearized sub-problems. Rabih et al. [6] presented a homogeneous interior point (HIP) method for the ED problem by approximating the network constraints through the DC load flow, and the transmission losses through the B-matrix loss formula. Lin et al. [7] analyzed the mixed integer OPF based on the interior point cutting plane method (IPCPM). Also, they presented a new base identification method based on the improvement of IPCPM to solve the problems of degenerate solutions and convex combination solutions that depends on the difference between nonzero element number of optimal solution and rank of coefficient matrix.

Dragan et al. [8] demonstrated the feasibility of using a fuzzy expert system, based on interactive fuzzy linear programming (FLP) to optimal power system rescheduling 
problem, incorporating the preventive redispatch. Their aim was to explore the feasibility of creating an intelligent power system rescheduling system. Pathom et al. [9] proposed the fuzzy-optimization approach for solving the (DED) under an uncertain deregulated power system. Nima and Hadi [10] presented a real-coded genetic algorithm (RCGA) with arithmetic-average-bound crossover (AABX) and hybrid mutation (HM) to solve the nonconvex ED problem. Through few recent years, ACO algorithms are employed to solve optimization problems in different fields with more accurate and efficiently solution compared with conventional and other modern optimization algorithms.

In this paper, an ACO-based algorithm is proposed to solve the non-smooth ED problem. The minimization of the total fuel generation costs is considered as an objective function with equality and inequality constraints.

\section{Problem formulation:}

The SCOD problem can express as a constrained optimization problem as:

$$
\operatorname{Min} f(x)
$$

subject to:

$$
\begin{gathered}
g(x)=0 \\
h(x) \leq 0
\end{gathered}
$$

Where, $f(x)$ is the objective function such as generators fuel costs, $g(x)$ represents the equality constraints, $h(x)$ represents the inequality constraints, and $x$ is the vector of the control variables that may be generator real power outputs.

In this paper, the objective function is the non-linear fuel cost of generators with the valve-point effects that appears in a rectified sinusoidal function introduce ripples in the heat- rate curves, that's a function in the generator real power output, which are defined as:

$$
\begin{aligned}
\operatorname{Min} F_{t} & =\sum_{i=1}^{N G} f_{i}\left(P G_{i}\right) \\
& \left.=\sum_{i=1}^{N G}\left(a_{i}+b_{i} P G_{i}+c_{i} P G_{i}^{2}+e_{i} \times \sin \left(f_{i} \times\left(P G_{i}^{\mathrm{min}}-P G_{i}\right)\right)\right)\right)
\end{aligned}
$$

The objective function (2) is subjected to the following constraints:

\section{a) Equality constraints}


- Power balance constraint

The generators real power output should be equal to the total load demand and transmission line losses as:

$$
\sum_{i=1}^{N G} P G_{i}=\sum_{j=1}^{N L} P D{ }_{j}+P_{\text {losses }}
$$

The power loss is expressed using the B's coefficient as [11]:

$$
P_{\text {losses }}=\sum_{i=1}^{N G} \sum_{j=1}^{N G} P_{i} B_{i j} P_{j}
$$

Where, $P_{i}$ and $P_{j}$ are the injected powers at buses $i$ and $j$, respectively.

\section{b) Inequality constraints}

- The generator real power output must be within the feasible limits as:

$$
P G_{i}^{\min } \leq P G_{i} \leq P G_{i}^{\max }
$$

- Power flow constraints

The power flow in each transmission line $\mathrm{k}$ must be less than the maximum permissible power flow in the line $\mathrm{k}$ as:

$\left|P F_{k}\right|=\left|D_{k, i} P G_{i}\right| \leq P F_{k}^{\max }$

\section{ACO algorithm:}

ACO algorithms were proposed by Dorigo [12-14]. A brief description of the ACO is presented as follows:

\subsection{Description of real ants:}

ACO algorithms are based on the behavior of real ants that are members of a family of social insects. However, a group of explorer ants leave the colony for finding the food source in a randomly directions where they marked their routes by laying a chemical substance on the ground. Other ants attractive to the route that has the largest amount of pheromone that decays with time. So that, a shorter route will be found that has a largest amount of pheromone than a longer route. Hence, they are found the shortest route between the nest and food source by indirect communication media that called pheromone that laid on the ground as a guide for another ants. Fig. 1 shows how the real ants can find the shortest path between nest or colony and food source. In Fig. 1(a), 
there are no obstacles between nest and food source. However, the shortest route is the straight line. If an obstacle is located on the route of ants to become two routes around the obstacle, some of ants choose the left side around the obstacle and the other will choose the right side as shown in Fig. 1(b). The pheromone laid on the left side will be concentrated than right side of obstacle because ants in the shortest path takes minimum time in leaving and returning for nest where they moves in the same speed. So, they will be laid a largest amount of pheromone than other ants on the other route. While, the other ants attractive to the shortest route. Hence, all ants in the colony will take the shortest route around the obstacle as shown in Fig. 1(c).

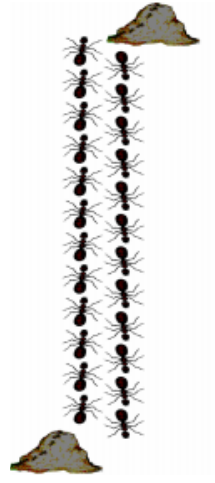

a.

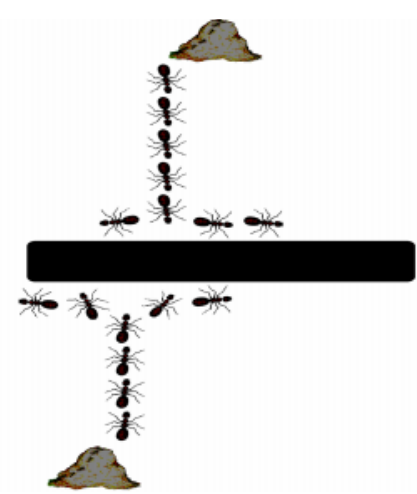

b.

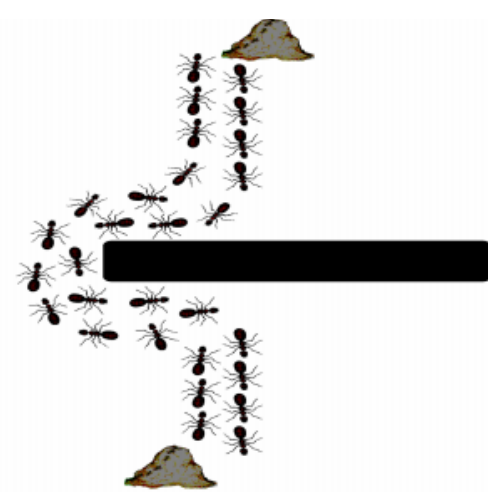

c.

Figure (1): Illustration of real ants behaviour

\subsection{Mathematical model of ACO algorithm:}

A random amount of pheromone is deposited in each rout after each ant completes its tour, anther antes attract to the shortest route according to the probabilistic transition rule that depends on the amount of pheromone deposited and a heuristic guide function as equal to the inverse of the distance between beginning and ending of each route. The probabilistic transition rule of ant $k$ to go from city $i$ to city $j$ can be expressed as in Traveling Salesman Problem (TSP) [14] as:

$$
P_{i j}^{k}(t)=\frac{\left[\tau_{i j}(t)\right]^{\alpha}\left[\eta_{i j}(t)\right]^{\beta}}{\sum_{q}\left[\tau_{i q}(t)\right]^{\alpha}\left[\eta_{i q}(t)\right]^{\beta}} ; j, q \in N_{i}^{k}
$$

If $\alpha=0$, the closest cities are more likely to be selected that corresponding to a classical greedy algorithm. On the contrary, if $\beta=0$, the probability will be depend on the 
pheromone trial only. These two parameters should be tuned with each other, Dorigo in [12] found experimentally the good values of $\alpha$ and $\beta$ are 1 and 5, respectively, $q$ is the cities that will be visited after city $i$. While, $N_{r}{ }^{k}$ is a tabu list in memory of ant that recodes the cities are visited to avoid stagnations. After each tour is completed, a local pheromone update is determined by each ant depending on the route of each ant as in Equation (8). After all ants attractive to the shortest route, a global pheromone update is considered to show the influence of the new addition deposits by the other ants that attractive to the best tour as shown in Equation (9).

$$
\begin{aligned}
& \tau_{i j}(t+1)=(1-\rho) \tau_{i j}(t)+\rho \tau_{\mathrm{o}} \\
& \tau_{i j}(t+1)=(1-\rho) \tau_{i j}(t)+\varepsilon \Delta \tau_{i j}(t)
\end{aligned}
$$

the amount of pheromone for elite path can be calculated as:

$$
\Delta \tau_{i j}(t)=1 / d_{\text {best }}
$$

\section{ACO algorithm for SCOD problem:}

ACO algorithm is applied to solve the SCOD problem as an optimization technique with equality and inequality constraints where artificial ants travels in search space to find the shortest route that having the strongest pheromone trail and a minimum cost function. The SCOD aims at minimizing the total cost of generation real output power (2) with equality and inequality constraints (Equations (3) - (6)). So, a heuristic guide function is the inverse of the individual cost of each ant that positioned in the reasonable limit of the control variable to the visibility of each ant. While, heuristic guide function of the problem is the inverse of the total costs at iteration $t+1$ as:

$$
\eta(t+1)=1 / \sum_{i=1}^{N G} f_{i}\left(P G_{i}\right)
$$

In GA, a chromosome is subdivided into genes, each gene represents a variable, consists of a binary string with length that depends on the boundary of the corresponding variable.

In ACO algorithm, a search space creates with dimensions of stages on number of control variables and states or the randomly distributed values of control variables with in a reasonable threshold. Artificial ant's leaves colony to search randomly in the search space based on the probability in (7) to complete a tour matrix that consists of the 
positions of ants with the same dimension of the search space. Then, tour matrix is applied on the objective function to find a heuristic guide function to find the best solution and update local and global pheromone to begin a next iteration. System parameters are adjusted by trail and error to find the best values of theses parameters.

The ACO algorithm can be applied to solve the SCOD problem using the following steps:

\section{Step 1: Initialization}

Insert the lower and upper boundaries of each control variable $\left(\mathrm{PG}^{\mathrm{min}}, \mathrm{PG}^{\mathrm{max}}\right)$, system parameters and create a search space with a dimensions of number of control variables (PG) and the length of randomly distributed values with the same dimension of the initial pheromone that contains elements with very small equal values to give all ants with the same chance of searching.

\section{Step 2: Provide first position}

Each ant is positioned on the initial state randomly with in the reasonable range of each control variable in a search space with one ant in each control variable in the length of randomly distributed values.

\section{Step 3: Transition rule}

Each ant decide to visit a next position in the range of other control variables according to the probability transition rule in Equation (7) that depends on the amount of pheromone deposited and the visibility that is the inverse of objective function (11). Where, the effect of pheromone and visibility on each other depends on the two parameters $\alpha$ and $\beta$.

\section{Step 4: Local pheromone updating}

Local updating pheromone is different from ant to other because each ant takes a different route. The initial pheromone of each ant is locally updated as in equation (8).

\section{Step 5: Fitness function}

After all ants attractive to the shortest path that having a strongest pheromone, the best solution of the objective function is obtained.

\section{Step 6: Global pheromone updating}

Amount of pheromone on the best tour becomes the strongest due to attractive of ants for this path. Moreover, the pheromone on the other paths is evaporated in time. 


\section{Step 7: Program termination}

The program will be terminated when the maximum iteration is reached or the best solution is obtained without the ants stagnations.

\section{Applications:}

\subsection{Test systems:}

Three standard test systems and a real power system are used to study the proposed technique for SCOD using an ACO algorithm. These standard systems are 5-bus [15], IEEE 14-bus and IEEE 30-bus test systems [16]. The 5-bus system has three generator units at buses 1, 2 and 5. The IEEE 14 bus system has two generators at buses 1 and 2. It is considered the first lines of the standard systems as most critical lines of these systems. The maximum permissible power flows of these critical lines are equal to 45, 150 and $65 \mathrm{MW}$ for 5-bus, 14-bus and 30 bus test systems, respectively. However the power flows of other lines in the three systems are preserved within their security limits. An application to a real power system at the West Delta Network (WDN) is a part of the Unified Egyptian Network (UEN) [17] as shown in Fig. 2.

The results that obtained are compared with those obtained using conventional optimization techniques namely linear programming (LP), fuzzy linear programming (FLP) and genetic algorithm (GA) techniques [15]. The best values of ACO algorithm parameters are $\alpha=1, \beta=8, \rho=0.5$ and $\varepsilon=5$.

Two different operation conditions are considered to obtain the SCOD, which are normal and emergency conditions.

The emergency conditions that may occur in the three test systems are:

a) Sudden increase in load demand.

b) Unexpected outage of one line.

c) Unexpected outage of units inside the generation plant.

\subsection{Results and comments:}

The results are obtained using ACO algorithm that processed using MatLab code version 7.1 that setup on a Pentium 4, 3.0 GHz PC, $0.99 \mathrm{~GB}$ of ram.

\subsubsection{Normal conditions:}


Tables 1, 2 and 3 shows a comparison between the results obtained using ACO algorithm and the previous results using conventional LP, the FLP and GA [14]. In theses tables, the ACO algorithm has the minimum generation cost compared with other techniques.

Table 4 shows a comparison between the results obtained using ACO algorithm and the results using conventional LP, the FLP and GA for WDN system with valve-point effects are taken into account. In this table, the ACO algorithm has the lowest generation cost compared with other optimization techniques. The computation time using ACO algorithm dependent on the system size and related to the number of control variables in Tables 1-4, the computation time is $2.1,2.25,2.28$ and 2.344 corresponding to $14,5,30$ and WDN systems that have 2, 3, 6 and 8 number of control variables, respectively.

Table (1): Comparison of various optimization methods for5-bus system (load=185 MW)

\begin{tabular}{|c|c|c|c|c|}
\hline Variable & LP & FLP & GA & ACO \\
\hline \hline $\mathrm{PG}_{1}$ & 90.2 & 78.9 & 90.2 & 90.15 \\
\hline $\mathrm{PG}_{2}$ & 34.8 & 61.7 & 35.8 & 35.17 \\
\hline $\mathrm{PG}_{5}$ & 60 & 44.4 & 59 & 59.68 \\
\hline $\mathrm{PF}_{1}$ & 45 & 34.6 & 44.98 & 44.94 \\
\hline Cost $\mathbf{( \$ h r )}$ & $\mathbf{3 8 0 . 7}$ & $\mathbf{3 9 1 . 7}$ & $\mathbf{3 7 4 . 1}$ & $\mathbf{3 7 3 . 8}$ \\
\hline Time (Sec.) & 0.55 & 0.66 & 0.99 & 2.25 \\
\hline
\end{tabular}

Table (2): Comparison of various optimization methods for14-bus system (load=260 MW)

\begin{tabular}{|c|c|c|c|c|}
\hline Variable & LP & FLP & GA & ACO \\
\hline $\mathrm{PG}_{1}$ & 208.1 & 196.8 & 208.1 & 208.01 \\
\hline $\mathrm{PG}_{2}$ & 51.86 & 63.2 & 51.9 & 51.99 \\
\hline $\mathrm{PF}_{1}$ & 150 & 140.4 & 149.96 & 149.92 \\
\hline Cost $\mathbf{\$} / \mathbf{h r})$ & $\mathbf{9 5 8 . 1}$ & $\mathbf{9 6 1 . 4}$ & $\mathbf{7 6 7 . 5}$ & $\mathbf{7 6 3 . 4}$ \\
\hline Time $($ Sec.) & 0.5 & 0.72 & 1.1 & 2.1 \\
\hline
\end{tabular}

Table (3): Comparison of various optimization methods for30-bus system (load=220 MW) 


\begin{tabular}{|c|c|c|c|c|}
\hline Variable & LP & FLP & GA & ACO \\
\hline PG1 & 10 & 52 & 49.1 & 59.73 \\
\hline PG2 & 80 & 62.3 & 65.6 & 55.89 \\
\hline PG3 & 39.4 & 28.9 & 21 & 40.81 \\
\hline PG4 & 10 & 16 & 23.7 & 17.5 \\
\hline PG5 & 30 & 25.5 & 16.5 & 27.12 \\
\hline PG6 & 50.6 & 35.3 & 44.1 & 18.95 \\
\hline PF1 & -0.251 & 30.94 & 28.07 & 37.75 \\
\hline Cost (\$/hr) & $\mathbf{8 7 1 . 9 3}$ & $\mathbf{8 7 9 . 2 2}$ & $\mathbf{6 8 5 . 1 3}$ & $\mathbf{6 5 4 . 9}$ \\
\hline Time (Sec.) & 0.6 & 1.1 & 1.75 & 2.28 \\
\hline
\end{tabular}

Table (4): Comparison of various optimization methods for WDN system (load=890 MW)

\begin{tabular}{|c|c|c|c|c|}
\hline Variable & LP & FLP & GA & $\mathrm{ACO}$ \\
\hline PG1 & 10 & 107.6704 & 70 & 62.218 \\
\hline PG2 & 10 & 123.2776 & 84.5 & 90.853 \\
\hline PG3 & 10 & 116.9019 & 81.2 & 83.74 \\
\hline PG4 & 250 & 104.6504 & 130.1 & 131.68 \\
\hline PG5 & 339.75 & 140.9867 & 192.5 & 170.08 \\
\hline PG6 & 250 & 74.3218 & 220.1 & 221.91 \\
\hline PG7 & 10 & 104.6519 & 56.2 & 66.42 \\
\hline PG8 & 10 & 117.2894 & 55.3 & 63.026 \\
\hline PF34 & 32.417 & 12.88 & 18.192 & 4.325 \\
\hline PF40 & 46.248 & 26.636 & 46.603 & 12.522 \\
\hline PF50 & 4.5307 & 12.535 & 9.1532 & 16.665 \\
\hline PF76 & 15.734 & 12.298 & 14.918 & 16.745 \\
\hline Cost $(\$ / h r)$ & 3149.3 & 3890.4 & 2909.8 & 2903.9 \\
\hline Time (Sec.) & 0.375 & 0.594 & 2.937 & 2.344 \\
\hline
\end{tabular}

\subsubsection{Emergency conditions:}




\section{- Sudden increase in the load demand:}

Tables 5 and 6 show the SCOD using the ACO algorithm for different loading conditions for the 5- bus and 14- bus test systems. In these tables, the power flows in the critical lines are kept within their limits, and the generation costs are increased according to the increasing of the load demand.

Table (5): SCOD solution using ACO algorithm for different loading conditions for 5bus system

\begin{tabular}{|c|c|c|c|c|c|c|}
\hline $\begin{array}{c}\text { Load (MW) } \\
\text { Variable }\end{array}$ & $\mathbf{1 5 0}$ & $\mathbf{1 7 0}$ & $\mathbf{1 8 5}$ & $\mathbf{2 0 0}$ & $\mathbf{2 2 0}$ & $\mathbf{2 3 0}$ \\
\hline $\mathrm{PG}_{1}$ & 82.74 & 86.87 & 90.15 & 92.36 & 92.63 & 93.06 \\
\hline $\mathrm{PG}_{2}$ & 14.51 & 27.93 & 35.17 & 49.76 & 69.83 & 79.67 \\
\hline $\mathrm{PG}_{3}$ & 52.75 & 55.2 & 59.68 & 57.88 & 57.54 & 57.27 \\
\hline $\mathrm{PF}_{1}$ & 44.83 & 44.86 & 44.94 & 43.92 & 40.397 & 38.88 \\
\hline Cost (\$/hr) & $\mathbf{2 9 5 . 1 2}$ & $\mathbf{3 4 0 . 0 1}$ & $\mathbf{3 7 3 . 8}$ & $\mathbf{4 0 9 . 6 2 6}$ & $\mathbf{4 6 0 . 2 4}$ & $\mathbf{4 8 5 . 9 2}$ \\
\hline
\end{tabular}

Table (6): SCOD solution using ACO algorithm for different loading conditions for 14bus system

\begin{tabular}{|c|c|c|c|c|c|c|}
\hline Load (MW) & $\mathbf{2 2 0}$ & $\mathbf{2 4 0}$ & $\mathbf{2 6 0}$ & $\mathbf{2 7 0}$ & $\mathbf{2 8 0}$ & $\mathbf{2 8 5}$ \\
\hline $\mathrm{PG}_{1}$ & 203.47 & 206.31 & 208.01 & 209.13 & 210.84 & 210.74 \\
\hline $\mathrm{PG}_{2}$ & 16.53 & 33.69 & 51.99 & 60.87 & 69.16 & 74.26 \\
\hline $\mathrm{PF}_{1}$ & 149.1 & 149.52 & 149.92 & 149.94 & 149.97 & 149.98 \\
\hline Cost $\mathbf{( \$ / h r})$ & $\mathbf{6 1 5 . 7 5}$ & $\mathbf{6 8 9 . 0 6}$ & $\mathbf{7 6 3 . 4}$ & $\mathbf{8 0 7 . 9 8}$ & $\mathbf{8 5 0 . 3}$ & $\mathbf{8 7 2 . 5 3}$ \\
\hline
\end{tabular}

Table 7 shows a comparison between the results obtained using ACO algorithm and GA for a WDN system for different loading conditions. In this table, the ACO algorithm has the minimum generation cost compared with GA.

Table (7): SCOD solution using ACO algorithm and GA for different loading conditions for the WDN system 


\begin{tabular}{|c|c|c|c|c|c|c|c|c|c|c|}
\hline Load(MW) & \multicolumn{2}{|c|}{$\mathbf{7 0 0}$} & \multicolumn{2}{c|}{$\mathbf{8 0 0}$} & \multicolumn{2}{c|}{$\mathbf{8 9 0}$} & \multicolumn{2}{c|}{$\mathbf{1 0 0 0}$} & \multicolumn{2}{c|}{$\mathbf{1 1 0 0}$} \\
\hline Algorithm & GA & ACO & GA & ACO & GA & ACO & GA & ACO & GA & ACO \\
\hline $\mathrm{PG}_{1}$ & 70 & 55.56 & 70 & 59.73 & 70 & 62.22 & 75.6 & 72.17 & 83.1 & 84.6 \\
\hline $\mathrm{PG}_{2}$ & 69.9 & 71.79 & 88.5 & 82.19 & 84.5 & 90.85 & 130 & 106.3 & 130 & 125.51 \\
\hline $\mathrm{PG}_{3}$ & 73.3 & 72.68 & 70 & 76.37 & 81.2 & 83.74 & 113.8 & 94.80 & 114.9 & 114.71 \\
\hline $\mathrm{PG}_{4}$ & 100 & 106.99 & 100 & 124.98 & 130.1 & 131.68 & 129.9 & 137.98 & 160.2 & 159.5 \\
\hline $\mathrm{PG}_{5}$ & 101.2 & 101.6 & 161.7 & 146.11 & 192.5 & 170.08 & 186.6 & 189.77 & 197 & 198 \\
\hline $\mathrm{PG}_{6}$ & 197.5 & 201.19 & 210.6 & 213.43 & 220.1 & 221.91 & 220 & 241.62 & 249.8 & 249.88 \\
\hline $\mathrm{PG}_{7}$ & 40 & 39.13 & 40 & 51.70 & 56.2 & 66.42 & 69.3 & 77.46 & 82.8 & 83.15 \\
\hline $\mathrm{PG}_{8}$ & 48.1 & 50.97 & 59.1 & 45.41 & 55.3 & 63.03 & 74.8 & 79.83 & 82.2 & 84.58 \\
\hline $\mathrm{PF}_{34}$ & 9.44 & 9.53 & 15.22 & 13.74 & 18.19 & 15.98 & 17.45 & 17.81 & 18.41 & 18.52 \\
\hline $\mathrm{PF}_{40}$ & 42.04 & 42.06 & 43.87 & 44.79 & 46.6 & 47.38 & 48.98 & 52.16 & 55.32 & 55.31 \\
\hline $\mathrm{PF}_{50}$ & 2.87 & 5.08 & 6.26 & 5.73 & 9.15 & 7.15 & 8.85 & 8.52 & 9.58 & 9.66 \\
\hline $\mathrm{PF}_{76}$ & 8.97 & 10.41 & 11.71 & 11.92 & 14.92 & 13.25 & 14.11 & 14.76 & 15.90 & 16.02 \\
\hline Cost(\$/hr) & $\mathbf{2 1 7 3}$ & $\mathbf{2 1 6 9 . 3}$ & $\mathbf{2 5 4 9 . 1}$ & $\mathbf{2 5 4 4 . 6}$ & $\mathbf{2 9 0 9 . 8}$ & $\mathbf{2 9 0 3 . 9}$ & $\mathbf{3 3 6 7 . 9}$ & $\mathbf{3 3 6 5}$ & $\mathbf{3 7 9 9 . 4}$ & $\mathbf{3 7 9 8 . 9}$ \\
\hline Time$($ Sec. & 3.063 & 2.219 & 2.891 & 2.266 & 2.937 & 2.344 & 2.922 & 2.343 & 3.031 & 3.462 \\
\hline
\end{tabular}

\section{- Unexpected outage of transmission line:}

Tables 8 and 9 show the SCOD computed using the ACO algorithm for different lines outage compared with the load flow (LF) using the Newton-Raphson method for 5-bus and 14-bus test systems. In these tables, overflows in the critical lines are removed using the ACO algorithm.

Table (8): SCOD solution for different line outage for 5-bus system (load=185 MW)

\begin{tabular}{|c|c|c|c|c|c|c|}
\hline \multirow{3}{*}{ Variables } & \multicolumn{6}{c|}{ Outage } \\
\cline { 2 - 7 } & \multicolumn{2}{|c|}{ Line 1 } & \multicolumn{2}{c|}{ Line 2 } & \multicolumn{2}{c|}{ Line 6 } \\
\cline { 2 - 7 } & $\mathrm{LF}$ & $\mathrm{ACO}$ & $\mathrm{LF}$ & $\mathrm{ACO}$ & LF & ACO \\
\hline $\mathrm{PG}_{1}$ & 90.15 & 59.98 & 90.15 & 59.89 & 90.15 & 85.61 \\
\hline $\mathrm{PG}_{2}$ & 35.17 & 69.10 & 35.17 & 69.24 & 35.17 & 40.12 \\
\hline $\mathrm{PG}_{3}$ & 59.68 & 55.92 & 59.68 & 55.87 & 59.68 & 59.27 \\
\hline $\mathrm{PF}_{1}$ & \multicolumn{2}{|c|}{ Outage } & $77.15^{*}$ & 44.78 & $49.46^{*}$ & 44.23 \\
\hline $\mathrm{PF}_{2}$ & $73.557^{*}$ & 44.735 & \multicolumn{2}{c|}{ Outage } & 31.65 & 26.35 \\
\hline * Denotes an overflow in transmission line, Where, the $\mathrm{PF}_{2}{ }^{\max }$ is $45 \mathrm{MW}$ \\
\hline
\end{tabular}

Table (9): SCOD solution for different line outage for 14-bus system (load=260 MW) 


\begin{tabular}{|c|c|c|c|c|c|c|}
\hline Line Outage & \multicolumn{2}{|c|}{7} & \multicolumn{2}{c|}{6} & \multicolumn{2}{c|}{10} \\
\hline \hline Algorithm & LF & ACO & LF & ACO & LF & ACO \\
\hline \hline $\mathrm{PG}_{1}$ & 208.01 & 185.63 & 208.01 & 203.45 & 208.01 & 204.28 \\
\hline $\mathrm{PG}_{2}$ & 51.99 & 74.37 & 51.99 & 56.55 & 51.99 & 55.72 \\
\hline $\mathrm{PF}_{1}$ & $166.49^{*}$ & 149.38 & $151.99^{*}$ & 148.46 & $151.45^{*}$ & 149.41 \\
\hline $\mathrm{PF}_{7}$ & \multicolumn{2}{|c|}{ Outage } & 47.016 & 28.204 & 98.379 & 94.251 \\
\hline
\end{tabular}

* Denotes an overflow in transmission line Where, Where, the $\mathrm{PF}_{7}{ }^{\mathrm{max}}$ is $100 \mathrm{MW}$

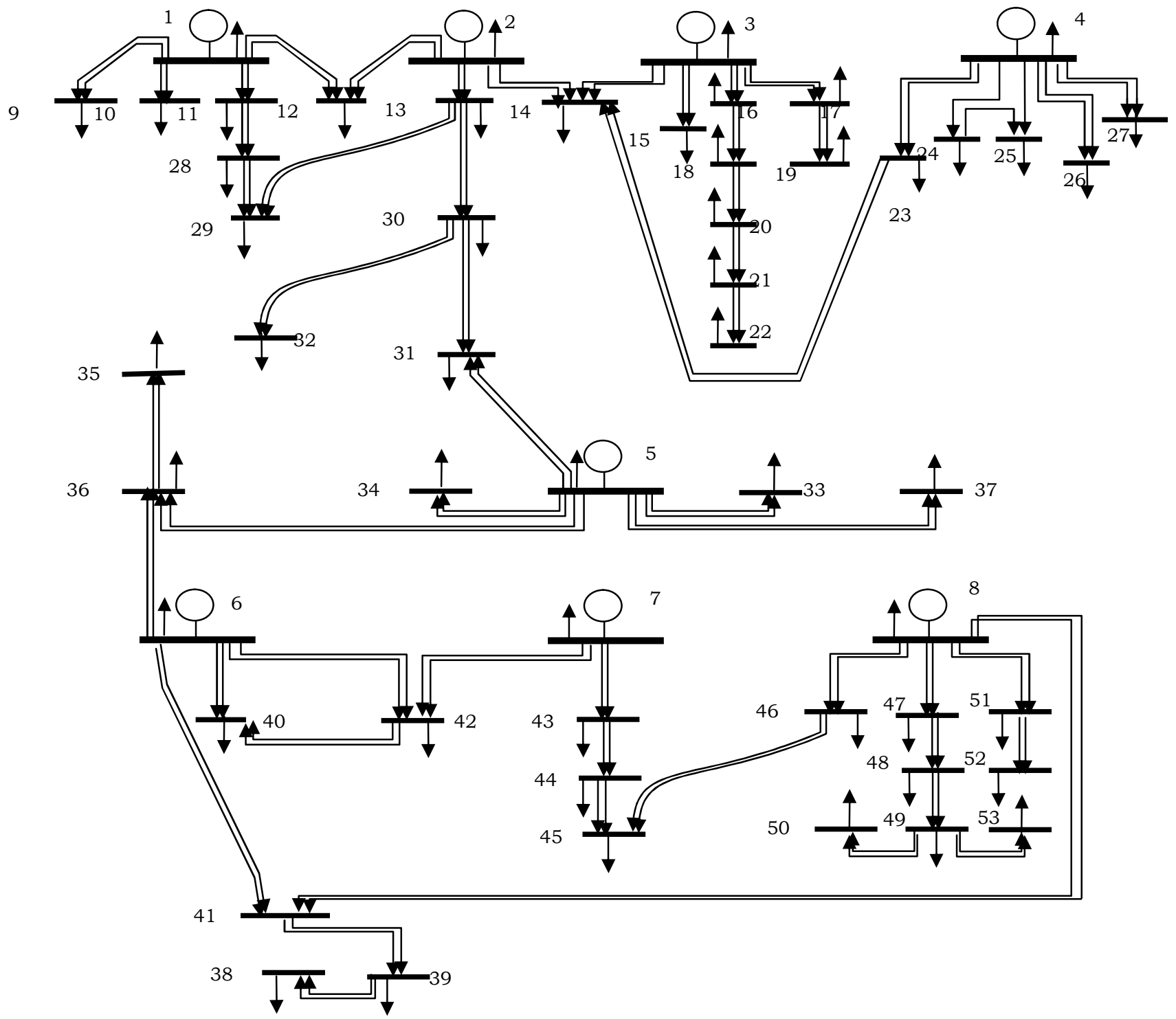

Figure (2): Single line diagram of the WDN system

Table 10 shows the SCOD computed using the ACO algorithm for different lines outage compared with the load flow (LF) using the Newton-Raphson method for WDN system. 
The power flows in all lines are kept within their permissible limits.

Table (10): SCOD solution for different line outage for WDN system (load=890 MW)

\begin{tabular}{|c|c|c|c|c|c|c|}
\hline Line Outage & \multicolumn{2}{|c|}{34} & \multicolumn{2}{|c|}{40} & \multicolumn{2}{|c|}{50} \\
\hline Technique & LF & $\mathrm{ACO}$ & LF & $\mathrm{ACO}$ & LF & $\mathrm{ACO}$ \\
\hline $\mathrm{PG}_{1}$ & 62.22 & 64.56 & 62.22 & 63.32 & 62.22 & 64.97 \\
\hline $\mathrm{PG}_{2}$ & 90.85 & 91.08 & 90.85 & 90.98 & 90.85 & 91.18 \\
\hline $\mathrm{PG}_{3}$ & 83.74 & 84.21 & 83.74 & 85.081 & 83.74 & 85.82 \\
\hline $\mathrm{PG}_{4}$ & 131.68 & 131.5 & 131.68 & 131.8 & 131.68 & 132.1 \\
\hline $\mathrm{PG}_{5}$ & 170.08 & 174.28 & 170.08 & 175.99 & 170.08 & 174.28 \\
\hline $\mathrm{PG}_{6}$ & 221.91 & 224.71 & 221.91 & 223.77 & 221.91 & 221.88 \\
\hline $\mathrm{PG}_{7}$ & 66.42 & 63.92 & 66.42 & 65.23 & 66.42 & 64.92 \\
\hline $\mathrm{PG}_{8}$ & 63.03 & 55.67 & 63.03 & 53.764 & 63.03 & 54.78 \\
\hline $\mathrm{PF}_{34}$ & \multicolumn{2}{|c|}{ Outage } & 79.49 & 16.23 & 82.23 & 16.41 \\
\hline $\mathrm{PF}_{40}$ & 40.74 & 47.21 & \multicolumn{2}{|c|}{ Outage } & 38.9 & 47.44 \\
\hline $\mathrm{PF}_{50}$ & 22.83 & 6.66 & 19.692 & 3.83 & \multicolumn{2}{|c|}{ Outage } \\
\hline $\mathrm{PF}_{76}$ & 5.384 & 13.015 & 5.387 & 13.02 & 5.38 & 13.01 \\
\hline
\end{tabular}

\section{- Unexpected outage of some units inside the generation plant:}

Figures 3 and 4 show the SCOD using ACO algorithm for different percentage outage of generation plants 1 and 3 for the 5-bus test system. From these figures, the power generation at bus $2\left(\mathrm{PG}_{2}\right)$ is increased largely according to an increase in the percentage outage of power generations 1 and 3 . While, the power generations at buses $3\left(\mathrm{PG}_{3}\right)$ and $1\left(\mathrm{PG}_{1}\right)$ are increased smally according to an increase in the percentage outage of power generations 1 and 3, respectively shown in Figures 3(a) and 4(a), as well as the generation costs are increased shown in Figures 3(b) and 4(b).

Figure 5 shows the SCOD using ACO algorithm for different percentage outage of generation plant 5 for the WDN system. From this figure, the power generation at other generation buses is increased according to an increase in the percentage outage of power generations 5, as well as the generation costs are increased. 


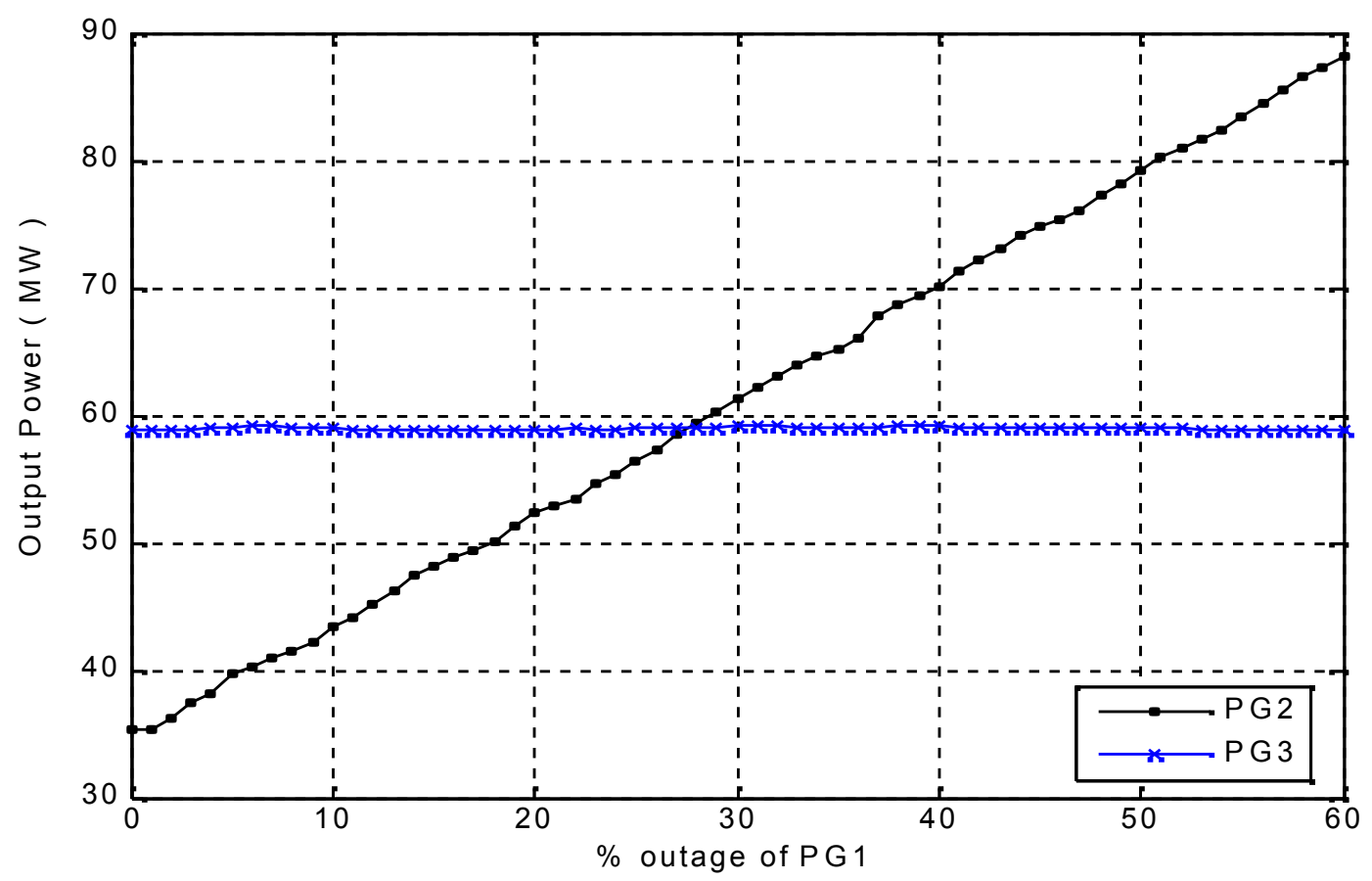

3. a) Variation of generation setting

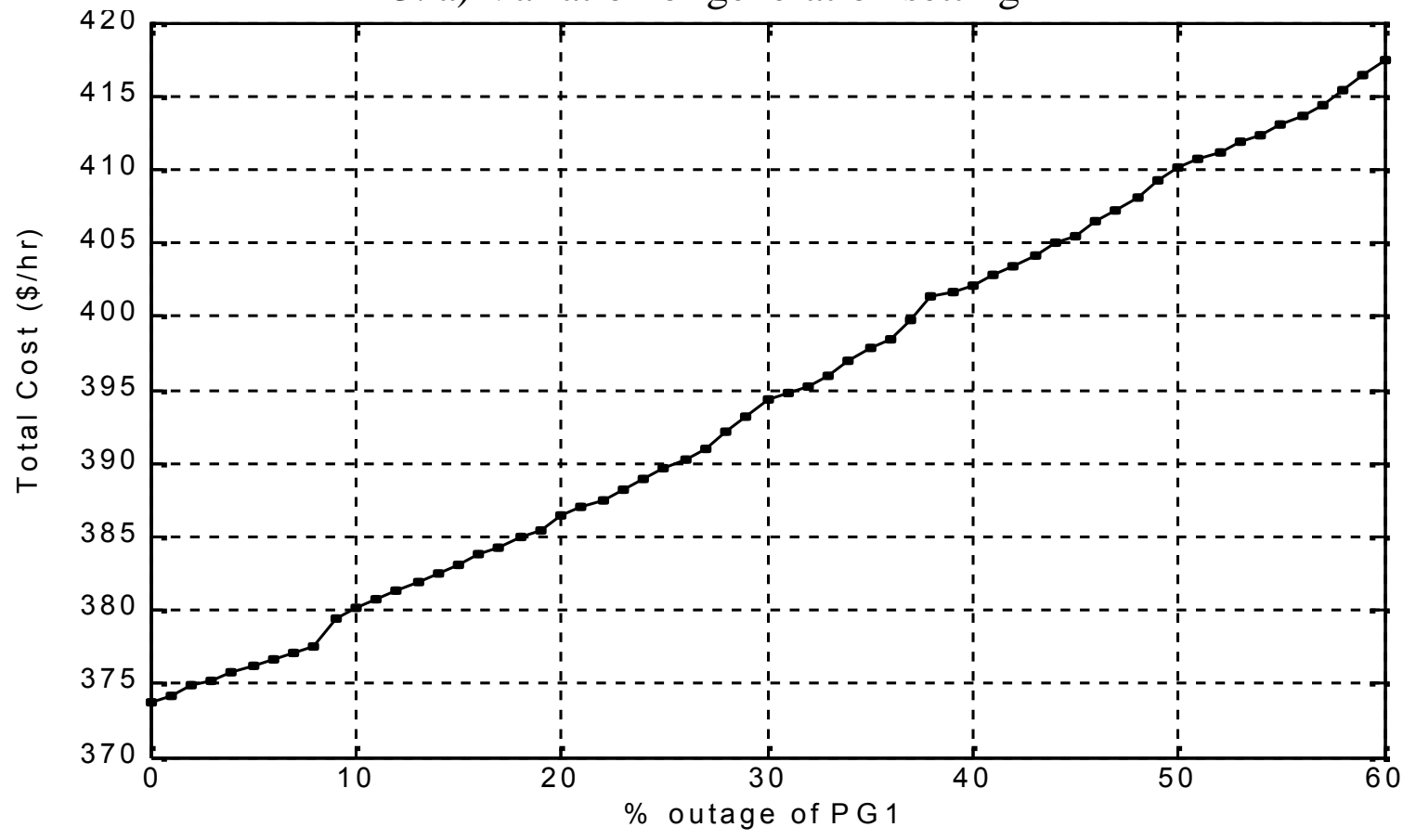

3. b) Variation of generation cost

Figure (3): The SCOD using ACO algorithm for different percentages outage of power generation 1 for 5-bus system 


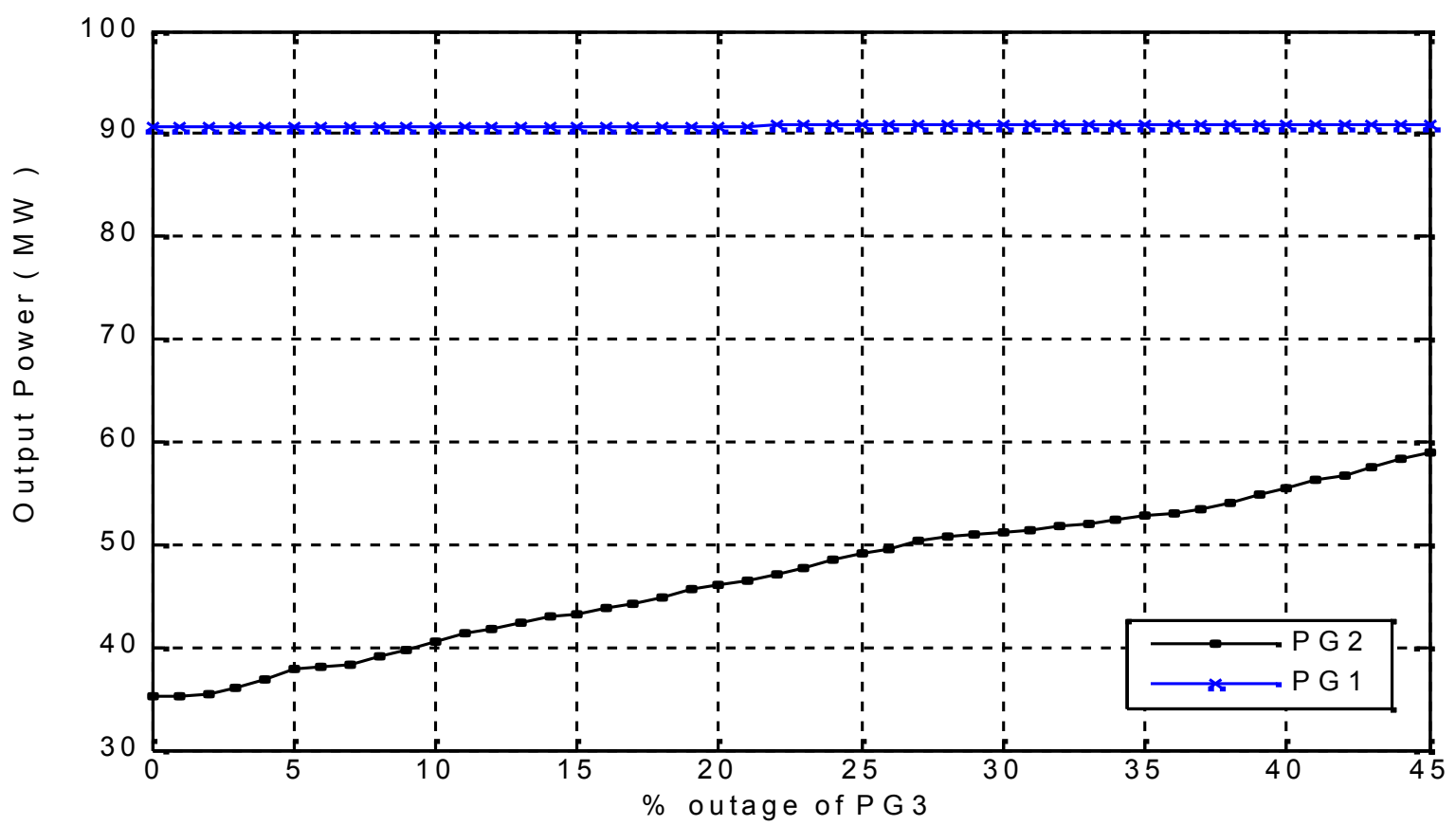

4. a) Variation of generation setting

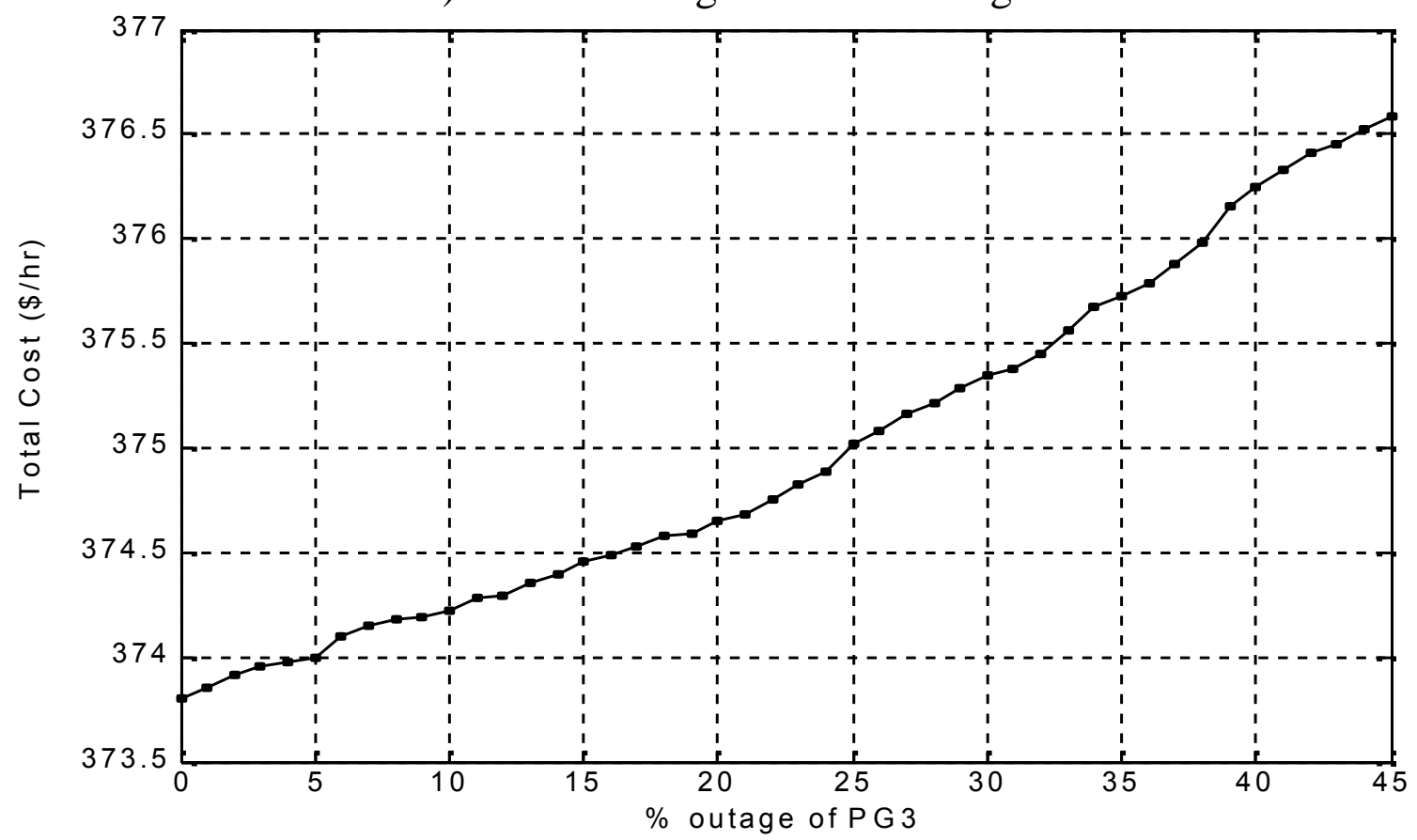

4. b) Variation of generation cost

Figure (4): The SCOD using ACO algorithm for different percentages outage of power generation 3 for 5-bus system 

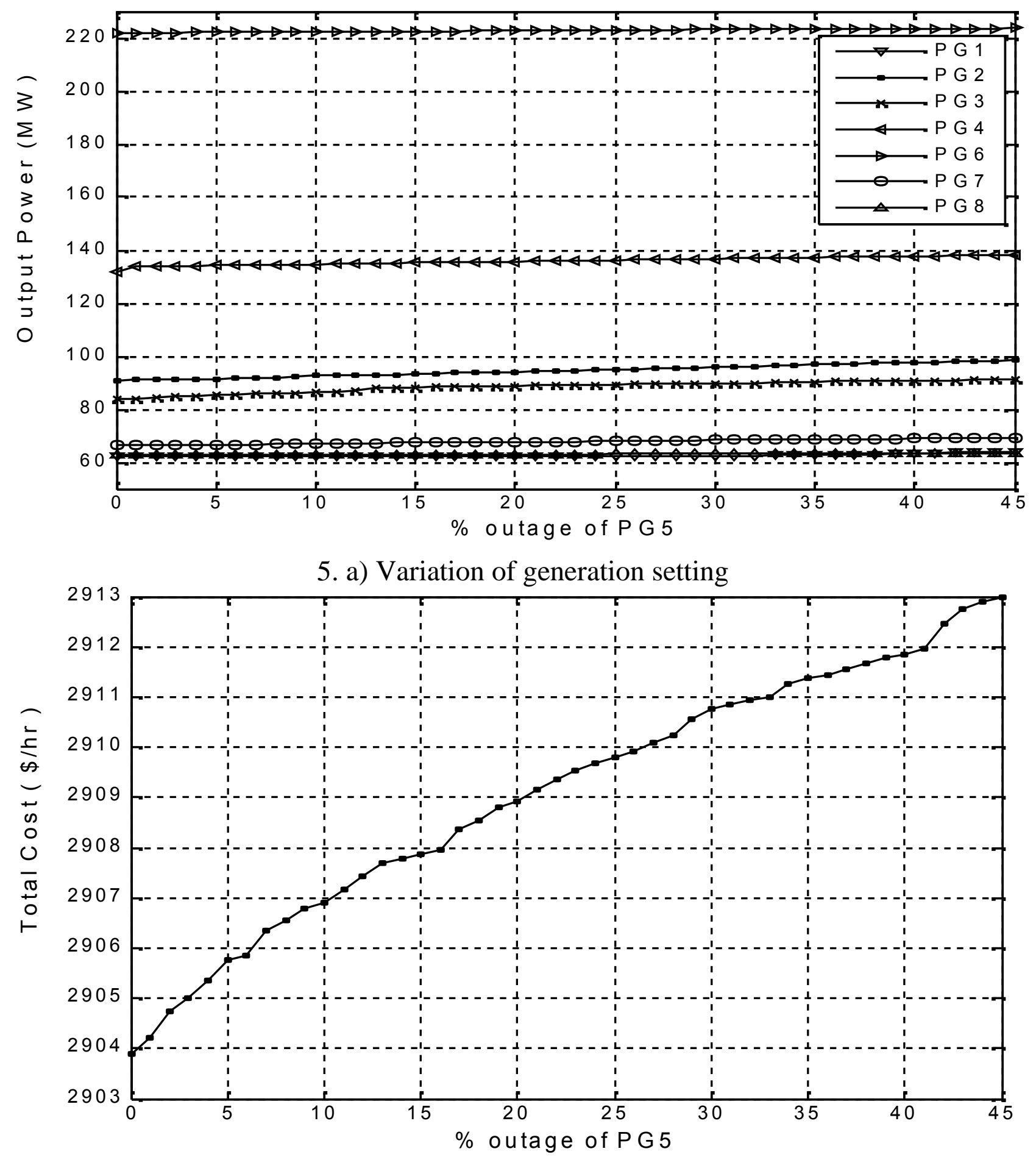

5. b) Variation of generation cost

Figure (5): The SCOD using ACO algorithm for different percentages outage of power generation 5 for WDN system 


\section{Conclusions:}

This paper presents an approach based on ACO algorithm to solve the problem of SCOD with equality and inequality constraints under normal and emergency conditions. The proposed algorithm has been tested on a three test systems and real actual system is the WDN system as a part of the Unified Egyptian Network (UEN), the results obtained are compared with other conventional LP, FLP and GA. The results show that, ACO algorithm leads to minimum generation costs for normal condition, while all the power flows in the critical lines are kept within their permissible limits. So, the proposed ACO algorithm gives more accurate and efficiently solution to remove the insecure operation at different emergency conditions. Therefore, the proposed algorithm represents a potential tool to aid the power system operators in the on-line environment.

\section{References:}

[1] M. Madrigal and V. H. Quintana, An Analytical Solution to the Economic Dispatch Problem, IEEE Power Engineering Review, Vol. 20, No. 9, P. 52-55, September 2000.

[2] Chun-Lung Chen and Nanming Chen, Direct Search Method for Solving Economic Dispatch Problem Considering Transmission Capacity Constraints, IEEE Trans. on Power Systems, Vol. 16, No. 4, P. 764-769, November 2001.

[3] P. Attaviriyanupap, H. Kita, E. Tanaka and J. Hasegawa, A Hybrid EP and SQP for Dynamic Economic Dispatch With Nonsmooth Fuel Cost Function, IEEE Trans. on Power Systems, Vol. 17, No. 2, P. 411-416, May 2002.

[4] Q. Xia, Y. H. Song, B. Zhang, C. Kang and N. Xiang, Dynamic Queuing Approach to Power System Short Term Economic and Security Dispatch, IEEE Trans. on Power Systems, Vol. 13, No. 2, P. 280-285, May 1998.

[5] X. Yan and V. H. Quintana, Improving An Interior-Point-Based OPF by Dynamic Adjustments of Step Sizes and Tolerances, IEEE Trans. on Power Systems, Vol. 14, No. 2, P. 709-717, May 1999.

[6] R. A. Jabr, A. H. Coonick and B. J. Cory, A study of the Homogeneous Algorithm for Dynamic Economic Dispatch with Network Constraints and Transmission Losses, IEEE Trans. on Power Systems, Vol. 15, No. 2, P. 605-611, May 2000.

[7] L. Liu, X. Wang, X. Ding and H. Chen, A Robust Approach to Optimal Power Flow With Discrete Variables, IEEE Trans. on Power Systems, Vol. 24, No. 3, P. 1182-1190, August 2009.

[8] D. Vlaisavljevic, M. B. Djukanovic, D. J. Sobajic and B. S. Babic, Fuzzy Linear Programming Based Optimal Power System Rescheduling Including Preventive 
Redispatch, IEEE Trans. on Power Systems, Vol. 14, No. 2, P. 525-531, May 1999.

[9] P. Attaviriyanupap, H. Kita, E. Tanaka and J. Hasegawa, A Fuzzy-Optimization Approach to Dynamic Economic Dispatch Considering Uncertainties, IEEE Trans. on Power Systems, Vol. 19, No. 3, P. 1299-1307, August 2004.

[10] N. Amjady and H. Nasiri-Rad, Nonconvex Economic Dispatch With AC Constraints by a New Real Coded Genetic Algorithm, IEEE Trans. on Power Systems, Vol. 24, No. 3, P. 1489-1502, August 2009.

[11] D. J. Kothari and J. S. Dhillon, Power System Optimization, Prentice Hall of India, Third edition, October 2007.

[12] M. Dorigo, Optimization, Learning and Natural Algorithms, Ph. D. dissertation, Dept. Electron., Politecnico di Milano, Milan, Italy, 1992.

[13] Dorigo M. and C. Blum, Ant Colony Optimization Theory: A Survey, Theoretical Computer Science, Vol. 344 (2-3), P. 243-278, 2005.

[14] D. M. and L. M. Gambardella, Ant Colonies for the Traveling Salesman Problem, BioSystems, Vol. 43, P. 73-81, 1997.

[15] A. A. Abou El-Ela, M. A. Bishr, R. A. F. Saleh and T. Fetouh, Security Constrained Optimal Dispatch Using Genetic Algorithms for Normal and Emergency Conditions, Engineering Research Journal (ERJ), Minoufiya University, Faculty of Engineering, Egypt, Vol. 28, No. 2, P. 9-14, April 2005.

[16] Washington University Website: www.ee.washington.edu/research/pstca/

[17] R. A. El-Sehiemy, Performance of Transmission Network Under Deregulated Electrical Power Systems, Ph. D. thesis, Minoufiya University, Faculty of Engineering, Egypt, August 2008.

\section{Nomenclatures:}

$\mathrm{F}_{\mathrm{t}} \quad$ The non-linear objective function of power generation cost Rotor speed $a_{i}, b_{i}$ and $c_{i}$ The coefficients of power generation cost function Conductance

$\mathrm{e}_{\mathrm{i}}$ and $\mathrm{f}_{\mathrm{i}} \quad$ The fuel cost coefficients of the $i$ th unit with valve-points effects

NG The number of generation buses

$\mathrm{PG}_{\mathrm{i}} \quad$ The power generation at bus $i$

$\mathrm{PD}_{\mathrm{j}} \quad$ The load demand at load bus $j$

NL The number of load buses

$P_{\text {losses }} \quad$ The total power losses in the system

$\mathrm{PG}_{\mathrm{i}}^{\mathrm{max}}$ The maximum limit of power generation at bus $i$

$\mathrm{PG}_{\mathrm{i}}{ }^{\text {min }}$ The minimum limit of power generation at bus $i$

$\mathrm{PF}_{\mathrm{k}} \quad$ The power flow in line $k$

$\mathrm{PF}_{\mathrm{k}}{ }^{\mathrm{max}}$ The maximum power flow in line $k$ 
$D_{k, i} \quad$ The distribution parameters of the power flows related to the power generations

$\tau_{\mathrm{ij}} \quad$ The pheromone trail deposited between city $i$ and $j$

$\eta_{\mathrm{ij}} \quad$ The visibility or sight and equal to the inverse of the distance or the transition cost between city $i$ and $j\left(\eta_{\mathrm{ij}}=1 / \mathrm{d}_{\mathrm{ij}}\right)$

$\alpha$ and $\beta \quad$ Two parameters that influence the relative weight of pheromone trail and heuristic guide function, respectively

$\mathrm{N}_{\mathrm{r}}^{\mathrm{k}} \quad$ A tabu list in memory of ant that recodes the cities are visited to avoid stagnations

$\rho \quad$ The pheromone evaporation constant

$\varepsilon \quad$ The elite path weighting constant

$d_{\text {bes }} \quad$ The shortest tour distance found as in TSP 